\title{
AN EXPLICIT STRONG CONVERGENCE ITERATIVE SCHEME FOR SOLVING EQUILIBRIUM PROBLEMS
}

\author{
Kanikar Muangchoo \\ Faculty of Science and Technology \\ Rajamangala University of Technology \\ Phra Nakhon (RMUTP), 1381 Pracharat 1 Road \\ Wongsawang, Bang Sue \\ Bangkok 10800, THAILAND
}

\begin{abstract}
Many methods have been introduced previously to solve the equilibrium problem, of which the extragradient method is efficient. In this paper, we introduce a modified version of the extragradient algorithm to figure out equilibrium in a real Hilbert space. The proposed scheme based on an inertial scheme and explicit step size rule. The method uses a monotonic step size rule based on local bifunction information rather than of its Lipschitz-type parameters or other line search technique. We also prove the convergence theorem of the given algorithm and discuss its applications in particular classes of equilibrium classes. Finally, several computational tests are shown to demonstrate the efficiency of our proposed algorithm.
\end{abstract}

AMS Subject Classification: $65 \mathrm{Y} 05,65 \mathrm{~K} 15,47 \mathrm{H} 05,47 \mathrm{H} 10$

Key Words: equilibrium problem; strong convergence; Lipschitz-type conditions; extragradient method; Hilbert space

\section{Introduction}

Let $\mathbb{K}$ be a non-empty, convex and closed subset of real Hilbert space $\mathbb{E}$ and $f: \mathbb{E} \times \mathbb{E} \rightarrow \mathbb{R}$ be a bifunction satisfying $f(x, x)=0$, for each $x \in \mathbb{K}$. A equilibrium problem (EP) for $f$ on $\mathbb{K}$ is defined as follows:

$$
\text { Find } \varsigma^{*} \in \mathbb{K} \text { such that } f\left(\varsigma^{*}, y\right) \geq 0, \forall y \in \mathbb{K} \text {. }
$$

Moreover, $\Sigma$ represented by the solution set of the problem (EP). The term

Received: October 27, 2020

(C) 2021 Academic Publications 
"equilibrium problem" introduced in 1992 by Muu and Oettli [1] and has been further investigated by Blum and Oettli [2]. The problem (EP) is also known as the Ky Fan inequality owing to his addition to this field [3]. In this article, we consider the problem of equilibrium by taking following conditions:

(h1) $f: \mathbb{E} \times \mathbb{E} \rightarrow \mathbb{R}$ is said to be (see $[2,4]$ ) pseudomonotone on $\mathbb{K}$ if

$$
f\left(y_{1}, y_{2}\right) \geq 0 \Longrightarrow f\left(y_{2}, y_{1}\right) \leq 0, \forall y_{1}, y_{2} \in \mathbb{K} .
$$

(h2) $f: \mathbb{E} \times \mathbb{E} \rightarrow \mathbb{R}$ is said to be Lipschitz-type continuous [5] on $\mathbb{K}$ if there exist $c_{1}, c_{2}>0$ such that

$$
\begin{aligned}
& f\left(y_{1}, y_{2}\right)+f\left(y_{2}, y_{3}\right)+c_{1}\left\|y_{1}-y_{2}\right\|^{2}+c_{2}\left\|y_{2}-y_{3}\right\|^{2} \\
& \geq f\left(y_{1}, y_{3}\right), \forall y_{1}, y_{2}, y_{3} \in \mathbb{K} .
\end{aligned}
$$

(h3) For any weakly convergent $\left\{y_{n}\right\} \subset \mathbb{K}$ to $y^{*}$ satisfies the following condition:

$$
\limsup _{n \rightarrow \infty} f\left(y_{n}, y\right) \leq f\left(y^{*}, y\right)
$$

(h4) $f(y, \cdot)$ is convex and sub-differentiable on $\mathbb{E}$ for $y \in \mathbb{E}$.

In particular, the problem of equilibrium is a general mathematical problem in the context that it unifies different mathematical problems, i.e., the fixed point problems, the vector and scalar minimization problems, variational inequalities, the complementarity problems, problems of the saddle point, Nash equilibrium problems in non-cooperative games as well as the inverse optimization problems $[2,6,1,7]$. Also, an extensive study on the nature of equilibria and the description of numerical algorithms for the problem (EP) can be contained in $[6,2,8,9]$ and others in $[10,11,12,13,14]$.

Meanwhile by using the Korpelevich extragradient method [15], Flam et al. [16] and Quoc et al. [17], was introduced the following method to solve the equilibrium problems involving pseudomonotone and Lipschitz-type bifunction:

$$
\left\{\begin{array}{l}
x_{n} \in \mathbb{K}, \\
y_{n}=\arg \min \left\{\chi f\left(x_{n}, y\right)+\frac{1}{2}\left\|x_{n}-y\right\|^{2}: y \in \mathbb{K}\right\}, \\
x_{n+1}=\arg \min \left\{\chi f\left(y_{n}, y\right)+\frac{1}{2}\left\|x_{n}-y\right\|^{2}: y \in \mathbb{K}\right\},
\end{array}\right.
$$

where $0<\chi<\min \left\{\frac{1}{2 c_{1}}, \frac{1}{2 c_{2}}\right\}$ and $c_{1}, c_{1}$ are Lipschitz-type constants. The methods in $[16,17]$ are often considered to as the extragradient method primarily as a result of Korpelevich in [15] to solve the the saddle point problems. 
It is important to note that existing methods involve constant step size that depends on the information of Lipschitz-type constants of the bifunction $[16,17,18,19]$. This can lead to some restriction in the sense of the application because the Lipschitz-type constants are generally unknown or complicated to evaluate. Recently, Vinh et al. [20] introduced modifications of the gradient method for solving pseudo-monotone equilibrium problems with the new step size rule. But the step size is fixed and may depend on the choice of initial step size in results in [20].

So, a natural question arises, i.e., "Is it possible to introduce new inertial strong strongly convergent extragradient-type method with monotone step size rule to solve problem (EP)"?

In this study, we give a positive answer to this question, i.e., the gradient method still set up a strong convergence sequence by using a monotonic step size rule for solving equilibrium problems associated with pseudomonotone functions. Motivated by the works of Censor et al. [21] and Hieu et al. [22] we introduce a new inertial extragradient-type method to solve the problem (EP) in the setting of an infinite-dimensional real Hilbert space. In particular, the main contributions in this paper are explained as follows: (i) In this paper, we introduce an inertial subgradient extragradient method with a monotone step size rule to solve the equilibrium problem in real Hilbert spaces. (ii) The applications of these results are studied to solve particular classes of equilibrium problems in a real Hilbert space.

\section{Preliminaries}

In this section, we consider some important lemmas and definitions which are useful to prove the convergence analysis of the proposed algorithm. A metric projection $P_{\mathbb{K}}(x)$ of $x \in \mathbb{E}$ onto a closed and convex subset $\mathbb{K}$ of $\mathbb{E}$ is defined by $P_{\mathbb{K}}(x)=\underset{y \in \mathbb{K}}{\arg \min }\{\|y-x\|\}$.

Lemma 2.1. ([23]) Let $P_{\mathbb{K}}: \mathbb{E} \rightarrow \mathbb{K}$ be a metric projection such that

(i) $\left\|y_{1}-P_{\mathbb{K}}\left(y_{2}\right)\right\|^{2}+\left\|P_{\mathbb{K}}\left(y_{2}\right)-y_{2}\right\|^{2} \leq\left\|y_{1}-y_{2}\right\|^{2}, y_{1} \in \mathbb{K}, y_{2} \in \mathbb{E}$.

(ii) $y_{3}=P_{\mathbb{K}}\left(y_{1}\right)$ if and only if $\left\langle y_{1}-y_{3}, y_{2}-y_{3}\right\rangle \leq 0, \forall y_{2} \in \mathbb{K}$.

(iii) $\left\|y_{1}-P_{\mathbb{K}}\left(y_{1}\right)\right\| \leq\left\|y_{1}-y_{2}\right\|, y_{2} \in \mathbb{K}, y_{1} \in \mathbb{E}$. 
Lemma 2.2. ([24]) Assume that $\Psi: \mathbb{K} \rightarrow \mathbb{R}$ is a sub-differentiable, lower semi-continuous and convex function on $\mathbb{K}$. Then, $x \in \mathbb{K}$ is said to be a minimizer of $\Psi$ if and only if $0 \in \partial \Psi(x)+N_{\mathbb{K}}(x)$, where $\partial \Psi(x)$ denote the sub-differential of $\Psi$ at $x \in \mathbb{K}$ and $N_{\mathbb{K}}(x)$ denote the normal cone on $\mathbb{K}$ at $x$.

Lemma 2.3. ([25]) Assume that a real sequence $\left\{r_{n}\right\}$ non-negative numbers satisfying $r_{n+1} \leq\left(1-\mho_{n}\right) r_{n}+\mho_{n} \partial_{n}$, for all $n \in \mathbb{N}$. Moreover, $\left\{\mho_{n}\right\} \subset(0,1)$ and $\left\{\tilde{\partial}_{n}\right\} \subset \mathbb{R}$ be two sequences such that $\lim _{n \rightarrow \infty} \mho_{n}=0, \sum_{n=1}^{\infty} \mho_{n}=$ $+\infty$ and $\lim \sup _{n \rightarrow \infty} \partial_{n} \leq 0$. Then, $\lim _{n \rightarrow \infty} r_{n}=0$.

Lemma 2.4. ([26]) Suppose that $\left\{r_{n}\right\}$ be a sequence of real numbers such that there exists a subsequence $\left\{n_{i}\right\}$ of $\{n\}$ such that $r_{n_{i}}<r_{n_{i+1}}$ for all $i \in \mathbb{N}$. Then, there is a non decreasing sequence $m_{k} \subset \mathbb{N}$ such that $m_{k} \rightarrow \infty$ as $k \rightarrow \infty$, and the following conditions are fullfilled by all (sufficiently large) numbers $k \in \mathbb{N}$ :

$$
r_{m_{k}} \leq r_{m_{k+1}} \text { and } r_{k} \leq r_{m_{k+1}} .
$$

In fact, $m_{k}=\max \left\{j \leq k: r_{j} \leq r_{j+1}\right\}$.

Lemma 2.5. ([27]) For all $y_{1}, y_{2} \in \mathbb{E}$ and $\precsim \in \mathbb{R}$, then subsequent inequalities hold.

(i) $\left\|ð y_{1}+(1-ð) y_{2}\right\|^{2}=ð\left\|y_{1}\right\|^{2}+(1-ð)\left\|y_{2}\right\|^{2}-ð(1-ð)\left\|y_{1}-y_{2}\right\|^{2}$.

(ii) $\left\|y_{1}+y_{2}\right\|^{2} \leq\left\|y_{1}\right\|^{2}+2\left\langle y_{2}, y_{1}+y_{2}\right\rangle$.

A normal cone of $\mathbb{K}$ upon $x \in \mathbb{K}$ is defined by

$$
N_{\mathbb{K}}(x)=\{z \in \mathbb{E}:\langle z, y-x\rangle \leq 0, \forall y \in \mathbb{K}\} .
$$

Let a convex function $\Psi: \mathbb{K} \rightarrow \mathbb{R}$ and subdifferential of $\Psi$ at $x \in \mathbb{K}$ is defined by

$$
\partial \Psi(x)=\{z \in \mathbb{E}: \Psi(y)-\Psi(x) \geq\langle z, y-x\rangle, \forall y \in \mathbb{K}\}
$$

\section{Main results}

We given main algorithm in this section that combines a monotonic step size rule and and inertial technique. The detailed method is is given as follows: 


\section{Algorithm 1}

SteP 0: Select $\alpha>0, \chi_{0}>0, \mu \in(0,1)$ and $x_{-1}, x_{0} \in \mathbb{K}$. Moreover, choose $\left\{\beta_{n}\right\} \subset(0,1)$ satisfies the conditions: $\lim _{n \rightarrow \infty} \beta_{n}=0$ and $\sum_{n=1}^{\infty} \beta_{n}=+\infty$.

STEP 1: Determine $\alpha_{n}$ such that

$$
0 \leq \alpha_{n} \leq \hat{\alpha_{n}} \text { and } \hat{\alpha_{n}}= \begin{cases}\min \left\{\frac{\alpha}{2}, \frac{\epsilon_{n}}{\left\|x_{n}-x_{n-1}\right\|}\right\} & \text { if } x_{n} \neq x_{n-1}, \\ \frac{\alpha}{2} & \text { otherwise }\end{cases}
$$

where $\epsilon_{n}=\circ\left(\beta_{n}\right)$ a positive sequence, i.e., $\lim _{n \rightarrow \infty} \frac{\epsilon_{n}}{\beta_{n}}=0$.

STEP 2: Determine $t_{n}=\left(1-\beta_{n}\right)\left[x_{n}+\alpha_{n}\left(x_{n}-x_{n-1}\right)\right]$.

STEP 3: Determine $y_{n}=\underset{y \in \mathbb{K}}{\arg \min }\left\{\chi_{n} f\left(t_{n}, y\right)+\frac{1}{2}\left\|t_{n}-y\right\|^{2}\right\}$. If $t_{n}=y_{n}$, then stop and $y_{n}$ is a solution.

STEP 4: Determine $\omega_{n} \in \partial_{2} f\left(t_{n}, y_{n}\right)$ and find a set

$$
\mathbb{E}_{n}=\left\{z \in \mathbb{E}:\left\langle t_{n}-\chi_{n} \omega_{n}-y_{n}, z-y_{n}\right\rangle \leq 0\right\} .
$$

STEP 5: Determine $x_{n+1}=\underset{y \in \mathbb{E}_{n}}{\arg \min }\left\{\chi_{n} f\left(y_{n}, y\right)+\frac{1}{2}\left\|t_{n}-y\right\|^{2}\right\}$.

STEP 6: Determine $\chi_{n+1}$ in the following way:

$$
\chi_{n+1}=\left\{\begin{array}{lr}
\min \left\{\chi_{n}, \frac{\mu\left\|t_{n}-y_{n}\right\|^{2}+\mu\left\|x_{n+1}-y_{n}\right\|^{2}}{2\left[f\left(t_{n}, x_{n+1}\right)-f\left(t_{n}, y_{n}\right)-f\left(y_{n}, x_{n+1}\right)\right]}\right\} \\
\text { if } r\left(t_{n}, x_{n+1}\right)-f\left(t_{n}, y_{n}\right)-f\left(y_{n}, x_{n+1}\right)>0, \\
\chi_{n} \quad \text { otherwise. }
\end{array}\right.
$$

Set $n:=n+1$ and move back to STEP 1 .

Lemma 3.1. Assume that conditions (h1)-(h4) are held and $\left\{x_{n}\right\}$ be a sequence generated by Algorithm 1 is bounded sequence.

Proof. By following Lemma 2.2, we obtain

$$
0 \in \partial_{2}\left\{\chi_{n} f\left(y_{n}, y\right)+\frac{1}{2}\left\|t_{n}-y\right\|^{2}\right\}\left(x_{n+1}\right)+N_{\mathbb{E}_{n}}\left(x_{n+1}\right) .
$$

For $\omega \in \partial f\left(y_{n}, x_{n+1}\right)$ there exists $\bar{\omega} \in N_{\mathbb{E}_{n}}\left(x_{n+1}\right)$ such that $\chi_{n} \omega+x_{n+1}-t_{n}+\bar{\omega}=$ 0 . It follows that

$$
\left\langle t_{n}-x_{n+1}, y-x_{n+1}\right\rangle=\chi_{n}\left\langle\omega, y-x_{n+1}\right\rangle+\left\langle\bar{\omega}, y-x_{n+1}\right\rangle, \forall y \in \mathbb{E}_{n} .
$$

Due to $\bar{\omega} \in N_{\mathbb{E}_{n}}\left(x_{n+1}\right)$, implies that $\left\langle\bar{\omega}, y-x_{n+1}\right\rangle \leq 0$, for all $y \in \mathbb{E}_{n}$. Thus, we 
have

$$
\left\langle t_{n}-x_{n+1}, y-x_{n+1}\right\rangle \leq \chi_{n}\left\langle\omega, y-x_{n+1}\right\rangle, \forall y \in \mathbb{E}_{n} .
$$

Moreover $\omega \in \partial f\left(y_{n}, x_{n+1}\right)$, we have

$$
f\left(y_{n}, y\right)-f\left(y_{n}, x_{n+1}\right) \geq\left\langle\omega, y-x_{n+1}\right\rangle, \forall y \in \mathbb{E} .
$$

Combining (4) and (5), we get

$$
\chi_{n} f\left(y_{n}, y\right)-\chi_{n} f\left(y_{n}, x_{n+1}\right) \geq\left\langle t_{n}-x_{n+1}, y-x_{n+1}\right\rangle, \forall y \in \mathbb{E}_{n} .
$$

Due to the description of $\mathbb{E}_{n}$, we have

$$
\chi_{n}\left\langle\omega_{n}, x_{n+1}-y_{n}\right\rangle \geq\left\langle t_{n}-y_{n}, x_{n+1}-y_{n}\right\rangle .
$$

Now, using $\omega_{n} \in \partial f\left(t_{n}, y_{n}\right)$, we obtain

$$
f\left(t_{n}, y\right)-f\left(t_{n}, y_{n}\right) \geq\left\langle\omega_{n}, y-y_{n}\right\rangle, \forall y \in \mathbb{E} .
$$

By letting $y=x_{n+1}$, we have

$$
f\left(t_{n}, x_{n+1}\right)-f\left(t_{n}, y_{n}\right) \geq\left\langle\omega_{n}, x_{n+1}-y_{n}\right\rangle, \forall y \in \mathbb{E} .
$$

Combining (7) and (8), we get

$$
\chi_{n}\left\{f\left(t_{n}, x_{n+1}\right)-f\left(t_{n}, y_{n}\right)\right\} \geq\left\langle t_{n}-y_{n}, x_{n+1}-y_{n}\right\rangle .
$$

By replacing $y=\varsigma^{*}$ in $(6)$, we get

$$
\chi_{n} f\left(y_{n}, \varsigma^{*}\right)-\chi_{n} f\left(y_{n}, x_{n+1}\right) \geq\left\langle t_{n}-x_{n+1}, \varsigma^{*}-x_{n+1}\right\rangle .
$$

Since $\varsigma^{*} \in E p(f, \mathbb{K})$, we have $f\left(\varsigma^{*}, y_{n}\right) \geq 0$. From the pseudomonotonicity of $f$, we get $f\left(y_{n}, \varsigma^{*}\right) \leq 0$. It follows from (10) that

$$
\left\langle t_{n}-x_{n+1}, x_{n+1}-\varsigma^{*}\right\rangle \geq \chi_{n} f\left(y_{n}, x_{n+1}\right) .
$$

From description of $\chi_{n+1}$, we get

$$
f\left(t_{n}, x_{n+1}\right)-f\left(t_{n}, y_{n}\right)-f\left(y_{n}, x_{n+1}\right) \leq \frac{\mu\left\|t_{n}-y_{n}\right\|^{2}+\mu\left\|x_{n+1}-y_{n}\right\|^{2}}{2 \chi_{n+1}}
$$

From (11) and (12), we obtain

$$
\begin{aligned}
\left\langle t_{n}-x_{n+1}, x_{n+1}-\varsigma^{*}\right\rangle \geq & \chi_{n}\left\{f\left(t_{n}, x_{n+1}\right)-f\left(t_{n}, y_{n}\right)\right\} \\
& -\frac{\mu \chi_{n}}{2 \chi_{n+1}}\left\|t_{n}-y_{n}\right\|^{2}-\frac{\mu \chi_{n}}{2 \chi_{n+1}}\left\|x_{n+1}-y_{n}\right\|^{2} .
\end{aligned}
$$


Combining (9) and (13), we have

$$
\begin{aligned}
\left\langle t_{n}-x_{n+1}, x_{n+1}-\varsigma^{*}\right\rangle \geq & \left\langle t_{n}-y_{n}, x_{n+1}-y_{n}\right\rangle \\
& -\frac{\mu \chi_{n}}{2 \chi_{n+1}}\left\|t_{n}-y_{n}\right\|^{2}-\frac{\mu \chi_{n}}{2 \chi_{n+1}}\left\|x_{n+1}-y_{n}\right\|^{2} .
\end{aligned}
$$

We have the given formula in place:

$$
\begin{gathered}
-2\left\langle t_{n}-x_{n+1}, x_{n+1}-\varsigma^{*}\right\rangle=-\left\|t_{n}-\varsigma^{*}\right\|^{2}+\left\|x_{n+1}-t_{n}\right\|^{2}+\left\|x_{n+1}-\varsigma^{*}\right\|^{2} . \\
2\left\langle y_{n}-t_{n}, y_{n}-x_{n+1}\right\rangle=\left\|t_{n}-y_{n}\right\|^{2}+\left\|x_{n+1}-y_{n}\right\|^{2}-\left\|t_{n}-x_{n+1}\right\|^{2} .
\end{gathered}
$$

Combining (14) and (15)-(16), we get

$$
\left\|x_{n+1}-\varsigma^{*}\right\|^{2} \leq\left\|t_{n}-\varsigma^{*}\right\|^{2}-\left(1-\frac{\mu \chi_{n}}{\chi_{n+1}}\right)\left\|t_{n}-y_{n}\right\|^{2}-\left(1-\frac{\mu \chi_{n}}{\chi_{n+1}}\right)\left\|x_{n+1}-y_{n}\right\|^{2} .
$$

From expression (3), we have

$$
\alpha_{n}\left\|x_{n}-x_{n-1}\right\| \leq \epsilon_{n}, \forall n \in \mathbb{N},
$$

and due to $\lim _{n \rightarrow \infty}\left(\frac{\epsilon_{n}}{\beta_{n}}\right)=0$ gives that

$$
\lim _{n \rightarrow \infty} \frac{\alpha_{n}}{\beta_{n}}\left\|x_{n}-x_{n-1}\right\| \leq \lim _{n \rightarrow \infty} \frac{\epsilon_{n}}{\beta_{n}}=0 .
$$

By definition of $\left\{t_{n}\right\}$, we get

$$
\begin{aligned}
\left\|t_{n}-\varsigma^{*}\right\| & =\left\|x_{n}+\alpha_{n}\left(x_{n}-x_{n-1}\right)-\beta_{n} x_{n}-\alpha_{n} \beta_{n}\left(x_{n}-x_{n-1}\right)-\varsigma^{*}\right\| \\
& =\left\|\left(1-\beta_{n}\right)\left(x_{n}-\varsigma^{*}\right)+\left(1-\beta_{n}\right) \alpha_{n}\left(x_{n}-x_{n-1}\right)-\beta_{n} \varsigma^{*}\right\| \\
& \leq\left(1-\beta_{n}\right)\left\|x_{n}-\varsigma^{*}\right\|+\left(1-\beta_{n}\right) \alpha_{n}\left\|x_{n}-x_{n-1}\right\|+\beta_{n}\left\|\varsigma^{*}\right\| \\
& \leq\left(1-\beta_{n}\right)\left\|x_{n}-\varsigma^{*}\right\|+\beta_{n} \Gamma_{1},
\end{aligned}
$$

where

$$
\left(1-\beta_{n}\right) \frac{\alpha_{n}}{\beta_{n}}\left\|x_{n}-x_{n-1}\right\|+\left\|\varsigma^{*}\right\| \leq \Gamma_{1} .
$$

Given $\chi_{n} \rightarrow \chi$, there exists a fixed number $\Upsilon \in(0,1-\mu)$ such that

$$
\lim _{n \rightarrow \infty}\left(1-\frac{\mu \chi_{n}}{\chi_{n+1}}\right)=1-\mu>\Upsilon>0 .
$$

Hence, these exists $N_{1} \in \mathbb{N}$ and

$$
\left(1-\frac{\mu \chi_{n}}{\chi_{n+1}}\right)>\Upsilon>0, \forall n \geq N_{1}
$$


From (17), we obtain

$$
\left\|x_{n+1}-\varsigma^{*}\right\|^{2} \leq\left\|t_{n}-\varsigma^{*}\right\|^{2}, \forall n \geq N_{1} .
$$

Combining expressions (20) and (22), we get

$$
\begin{aligned}
\left\|x_{n+1}-\varsigma^{*}\right\| & \leq\left(1-\beta_{n}\right)\left\|x_{n}-\varsigma^{*}\right\|+\beta_{n} \Gamma_{1} \\
& \leq \max \left\{\left\|x_{n}-\varsigma^{*}\right\|, \Gamma_{1}\right\} \\
& \vdots \\
& \leq \max \left\{\left\|x_{N_{1}}-\varsigma^{*}\right\|, \Gamma_{1}\right\} .
\end{aligned}
$$

Thus, we can deduce that the sequence $\left\{x_{n}\right\}$ is bounded.

Theorem 3.1. Assume that $\left\{x_{n}\right\}$ be a sequence generated by Algorithm 1 and the conditions (h1)-(h4) are hold. Then, $\left\{x_{n}\right\}$ strongly converges to $\varsigma^{*}$. Moreover, $P_{E P(f, \mathbb{K})}(0)=\varsigma^{*}$.

Proof. By using (20) we have

$$
\begin{aligned}
\left\|t_{n}-\varsigma^{*}\right\|^{2} & \leq\left(1-\beta_{n}\right)^{2}\left\|x_{n}-\varsigma^{*}\right\|^{2}+\beta_{n}^{2} \Gamma_{1}^{2}+2 \Gamma_{1} \beta_{n}\left(1-\beta_{n}\right)\left\|x_{n}-\varsigma^{*}\right\| \\
& \leq\left\|x_{n}-\varsigma^{*}\right\|^{2}+\beta_{n}\left[\beta_{n} \Gamma_{1}^{2}+2 \Gamma_{1}\left(1-\beta_{n}\right)\left\|x_{n}-\varsigma^{*}\right\|\right] \\
& \leq\left\|x_{n}-\varsigma^{*}\right\|^{2}+\beta_{n} \Gamma_{2},
\end{aligned}
$$

for some $\Gamma_{2}>0$. From (17) and (24), we get

$$
\begin{aligned}
\left\|x_{n+1}-\varsigma^{*}\right\|^{2} \leq & \left\|x_{n}-\varsigma^{*}\right\|^{2}+\beta_{n} \Gamma_{2} \\
& -\left(1-\frac{\mu \chi_{n}}{\chi_{n+1}}\right)\left\|t_{n}-y_{n}\right\|^{2}-\left(1-\frac{\mu \chi_{n}}{\chi_{n+1}}\right)\left\|x_{n+1}-y_{n}\right\|^{2} .
\end{aligned}
$$

Due to the Lipschitz-continuity and pseudo-monotonicity of the bifunction $f$ implies that the solution set $\Sigma$ is a closed and convex set (for more details see $[17,18])$. Now, from $\varsigma^{*}=P_{\Sigma}(0)$ and by using Lemma 2.1 (ii), we have

$$
\left\langle 0-\varsigma^{*}, y-\varsigma^{*}\right\rangle \leq 0, \forall y \in \Sigma .
$$

The rest of the proof is divided into the following two parts:

Case 1: Assume that there is a fixed number $N_{2} \in \mathbb{N}\left(N_{2} \geq N_{1}\right)$ such that

$$
\left\|x_{n+1}-\varsigma^{*}\right\| \leq\left\|x_{n}-\varsigma^{*}\right\|, \quad \forall n \geq N_{2} .
$$


Thus, above implies that $\lim _{n \rightarrow \infty}\left\|x_{n}-\varsigma^{*}\right\|$ exists and let $\lim _{n \rightarrow \infty}\left\|x_{n}-\varsigma^{*}\right\|=l$. From the expression (25), we have

$$
\begin{aligned}
& \left(1-\frac{\mu \chi_{n}}{\chi_{n+1}}\right)\left\|t_{n}-y_{n}\right\|^{2}+\left(1-\frac{\mu \chi_{n}}{\chi_{n+1}}\right)\left\|x_{n+1}-y_{n}\right\|^{2} \\
& \leq\left\|x_{n}-\varsigma^{*}\right\|^{2}+\beta_{n} \Gamma_{2}-\left\|x_{n+1}-\varsigma^{*}\right\|^{2} .
\end{aligned}
$$

Due to the existence of limit of the sequence $\left\|x_{n}-\varsigma^{*}\right\|$ and $\beta_{n} \rightarrow 0$, we conclude that

$$
\left\|t_{n}-y_{n}\right\| \rightarrow 0 \text { and }\left\|x_{n+1}-y_{n}\right\| \rightarrow 0 \quad \text { as } n \rightarrow \infty .
$$

It continues from (29) that

$$
\lim _{n \rightarrow \infty}\left\|t_{n}-x_{n+1}\right\| \leq \lim _{n \rightarrow \infty}\left\|t_{n}-y_{n}\right\|+\lim _{n \rightarrow \infty}\left\|y_{n}-x_{n+1}\right\|=0 .
$$

Next, we need to compute

$$
\begin{aligned}
\left\|t_{n}-x_{n}\right\| & =\left\|x_{n}+\alpha_{n}\left(x_{n}-x_{n-1}\right)-\beta_{n}\left[x_{n}+\alpha_{n}\left(x_{n}-x_{n-1}\right)\right]-x_{n}\right\| \\
& \leq \alpha_{n}\left\|x_{n}-x_{n-1}\right\|+\beta_{n}\left\|x_{n}\right\|+\alpha_{n} \beta_{n}\left\|x_{n}-x_{n-1}\right\| \\
& =\beta_{n} \frac{\alpha_{n}}{\beta_{n}}\left\|x_{n}-x_{n-1}\right\|+\beta_{n}\left\|x_{n}\right\|+\beta_{n}^{2} \frac{\alpha_{n}}{\beta_{n}}\left\|x_{n}-x_{n-1}\right\| \longrightarrow 0 .
\end{aligned}
$$

The above is providing that

$$
\lim _{n \rightarrow \infty}\left\|x_{n}-x_{n+1}\right\| \leq \lim _{n \rightarrow \infty}\left\|x_{n}-t_{n}\right\|+\lim _{n \rightarrow \infty}\left\|t_{n}-x_{n+1}\right\|=0 .
$$

The above-mentioned details specifies that the sequences $\left\{t_{n}\right\}$ and $\left\{y_{n}\right\}$ are bounded. Due to the reflexivity of $\mathbb{E}$ and the boundedness of the sequence $\left\{x_{n}\right\}$ assurances that there have a $\left\{x_{n_{k}}\right\}$ subsequence such that $\left\{x_{n_{k}}\right\} \rightarrow \hat{x} \in \mathbb{E}$ as $k \rightarrow \infty$. Next, we have to prove that $\hat{x} \in \Sigma$. Due to the inequality (6), we have

$$
\chi_{n_{k}} f\left(y_{n_{k}}, y\right) \geq \chi_{n_{k}} f\left(y_{n_{k}}, x_{n_{k+1}}\right)+\left\langle t_{n_{k}}-x_{n_{k+1}}, y-x_{n_{k+1}}\right\rangle .
$$

From expression (12), we get

$$
\begin{aligned}
\chi_{n_{k}} f\left(y_{n_{k}}, x_{n_{k}+1}\right) \geq & \chi_{n_{k}}\left\{f\left(t_{n_{k}}, x_{n_{k}+1}\right)-f\left(t_{n_{k}}, y_{n_{k}}\right)\right\} \\
& -\frac{\mu \chi_{n_{k}}}{2 \chi_{n_{k}+1}}\left\|t_{n_{k}}-y_{n_{k}}\right\|^{2}-\frac{\mu \chi_{n_{k}}}{2 \chi_{n_{k}+1}}\left\|x_{n_{k}+1}-y_{n_{k}}\right\|^{2} .
\end{aligned}
$$

We also seen from the last two expressions that

$$
\begin{aligned}
& \chi_{n_{k}} f\left(y_{n_{k}}, y\right) \\
& \geq \chi_{n_{k}}\left\{f\left(t_{n_{k}}, x_{n_{k}+1}\right)-f\left(t_{n_{k}}, y_{n_{k}}\right)\right\}+\left\langle t_{n_{k}}-x_{n_{k+1}}, y-x_{n_{k+1}}\right\rangle
\end{aligned}
$$




$$
-\frac{\mu \chi_{n_{k}}}{2 \chi_{n_{k}+1}}\left\|t_{n_{k}}-y_{n_{k}}\right\|^{2}-\frac{\mu \chi_{n_{k}}}{2 \chi_{n_{k}+1}}\left\|x_{n_{k}+1}-y_{n_{k}}\right\|^{2}
$$

where $y$ is an arbitrary element in $\mathbb{E}_{n}$. From $(29),(30),(31),(32)$ and $\left\{x_{n}\right\}$ that the right side goes to zero. From $\chi_{n_{k}}>0$, the condition (h3) and $x_{n_{k}} \rightarrow \hat{x}$, we have

$$
0 \leq \limsup _{k \rightarrow \infty} f\left(y_{n_{k}}, y\right) \leq f(\hat{x}, y), \forall y \in \mathbb{E}_{n}
$$

The above implies that $f(\hat{x}, y) \geq 0, \forall y \in \mathbb{K}$, and hence $\hat{x} \in \Sigma$. It consider that

$$
\begin{aligned}
& \lim _{n \rightarrow \infty}\left\langle\varsigma^{*}, \varsigma^{*}-x_{n}\right\rangle \\
& =\limsup _{k \rightarrow \infty}\left\langle\varsigma^{*}, \varsigma^{*}-x_{n_{k}}\right\rangle=\left\langle\varsigma^{*}, \varsigma^{*}-\hat{x}\right\rangle \leq 0 .
\end{aligned}
$$

We can evaluate the following owing to $\lim _{n \rightarrow \infty}\left\|x_{n+1}-x_{n}\right\|=0$

$$
\begin{aligned}
& \limsup _{n \rightarrow \infty}\left\langle\varsigma^{*}, \varsigma^{*}-x_{n+1}\right\rangle \\
& \leq \limsup _{k \rightarrow \infty}\left\langle\varsigma^{*}, \varsigma^{*}-x_{n}\right\rangle+\limsup _{k \rightarrow \infty}\left\langle\varsigma^{*}, x_{n}-x_{n+1}\right\rangle \leq 0 .
\end{aligned}
$$

From expression (19), we can write

$$
\begin{aligned}
\| & t_{n}-\varsigma^{*} \|^{2} \\
= & \left\|x_{n}+\alpha_{n}\left(x_{n}-x_{n-1}\right)-\beta_{n} x_{n}-\alpha_{n} \beta_{n}\left(x_{n}-x_{n-1}\right)-\varsigma^{*}\right\|^{2} \\
= & \left\|\left(1-\beta_{n}\right)\left(x_{n}-\varsigma^{*}\right)+\left(1-\beta_{n}\right) \alpha_{n}\left(x_{n}-x_{n-1}\right)-\beta_{n} \varsigma^{*}\right\|^{2} \\
\leq & \left\|\left(1-\beta_{n}\right)\left(x_{n}-\varsigma^{*}\right)+\left(1-\beta_{n}\right) \alpha_{n}\left(x_{n}-x_{n-1}\right)\right\|^{2}+2 \beta_{n}\left\langle-\varsigma^{*}, t_{n}-\varsigma^{*}\right\rangle \\
= & \left(1-\beta_{n}\right)^{2}\left\|x_{n}-\varsigma^{*}\right\|^{2}+\left(1-\beta_{n}\right)^{2} \alpha_{n}^{2}\left\|x_{n}-x_{n-1}\right\|^{2} \\
& +2 \alpha_{n}\left(1-\beta_{n}\right)^{2}\left\|x_{n}-\varsigma^{*}\right\|\left\|x_{n}-x_{n-1}\right\| \\
& +2 \beta_{n}\left\langle-\varsigma^{*}, t_{n}-x_{n+1}\right\rangle+2 \beta_{n}\left\langle-\varsigma^{*}, x_{n+1}-\varsigma^{*}\right\rangle \\
\leq & \left(1-\beta_{n}\right)\left\|x_{n}-\varsigma^{*}\right\|^{2}+\alpha_{n}^{2}\left\|x_{n}-x_{n-1}\right\|^{2}+2 \alpha_{n}\left(1-\beta_{n}\right)\left\|x_{n}-\varsigma^{*}\right\| \\
& \left\|x_{n}-x_{n-1}\right\|+2 \beta_{n}\left\|\varsigma^{*}\right\|\left\|t_{n}-x_{n+1}\right\|+2 \beta_{n}\left\langle-\varsigma^{*}, x_{n+1}-\varsigma^{*}\right\rangle \\
= & \left(1-\beta_{n}\right)\left\|x_{n}-\varsigma^{*}\right\|^{2}+\beta_{n}\left[\alpha_{n}\left\|x_{n}-x_{n-1}\right\| \frac{\alpha_{n}}{\beta_{n}}\left\|x_{n}-x_{n-1}\right\|\right. \\
& +2\left(1-\beta_{n}\right)\left\|x_{n}-\varsigma^{*}\right\| \frac{\alpha_{n}}{\beta_{n}}\left\|x_{n}-x_{n-1}\right\| \\
& \left.+2\left\|\varsigma^{*}\right\|\left\|t_{n}-x_{n+1}\right\|+2\left\langle\varsigma^{*}, \varsigma^{*}-x_{n+1}\right\rangle\right] .
\end{aligned}
$$


From expressions (22) and (39), we get

$$
\begin{aligned}
& \left\|x_{n+1}-\varsigma^{*}\right\|^{2} \\
& \leq\left(1-\beta_{n}\right)\left\|x_{n}-\varsigma^{*}\right\|^{2}+\beta_{n}\left[\alpha_{n}\left\|x_{n}-x_{n-1}\right\| \frac{\alpha_{n}}{\beta_{n}}\left\|x_{n}-x_{n-1}\right\|\right. \\
& \quad+2\left(1-\beta_{n}\right)\left\|x_{n}-\varsigma^{*}\right\| \frac{\alpha_{n}}{\beta_{n}}\left\|x_{n}-x_{n-1}\right\| \\
& \left.\quad+2\left\|\varsigma^{*}\right\|\left\|t_{n}-x_{n+1}\right\|+2\left\langle\varsigma^{*}, \varsigma^{*}-x_{n+1}\right\rangle\right] .
\end{aligned}
$$

By (30), (38), (40) and applying Lemma 2.3, conclude that $\left\|x_{n}-\varsigma^{*}\right\| \rightarrow 0$ as $n \rightarrow \infty$.

Case 2: Assume that there is a subsequence $\left\{n_{i}\right\}$ of $\{n\}$,

$$
\left\|x_{n_{i}}-\varsigma^{*}\right\| \leq\left\|x_{n_{i+1}}-\varsigma^{*}\right\|, \forall i \in \mathbb{N} .
$$

Thus, by Lemma 2.4, is a sequence $\left\{m_{k}\right\} \subset \mathbb{N}$ as $\left\{m_{k}\right\} \rightarrow \infty$, $\left\|x_{m_{k}}-\varsigma^{*}\right\| \leq\left\|x_{m_{k+1}}-\varsigma^{*}\right\|$ and $\left\|x_{k}-\varsigma^{*}\right\| \leq\left\|x_{m_{k+1}}-\varsigma^{*}\right\|$, for all $k \in \mathbb{N}$.

Similar to Case 1, expression (28) provides that

$$
\begin{aligned}
& \left(1-\frac{\mu \chi_{m_{k}}}{\chi_{m_{k}+1}}\right)\left\|t_{m_{k}}-y_{m_{k}}\right\|^{2}+\left(1-\frac{\mu \chi_{m_{k}}}{\chi_{m_{k}+1}}\right)\left\|x_{m_{k}+1}-y_{m_{k}}\right\|^{2} \\
& \leq\left\|x_{m_{k}}-\varsigma^{*}\right\|^{2}+\beta_{m_{k}} \Gamma_{2}-\left\|x_{m_{k}+1}-\varsigma^{*}\right\|^{2} .
\end{aligned}
$$

Due to $\beta_{m_{k}} \rightarrow 0$, we deduce the following:

$$
\lim _{k \rightarrow \infty}\left\|t_{m_{k}}-y_{m_{k}}\right\|=\lim _{k \rightarrow \infty}\left\|x_{m_{k}+1}-y_{m_{k}}\right\|=0 .
$$

It follows that

$$
\lim _{k \rightarrow \infty}\left\|x_{m_{k+1}}-t_{m_{k}}\right\| \leq \lim _{k \rightarrow \infty}\left\|x_{m_{k+1}}-y_{m_{k}}\right\|+\lim _{k \rightarrow \infty}\left\|y_{m_{k}}-t_{m_{k}}\right\|=0 .
$$

Next, we have to evaluate

$$
\begin{aligned}
& \left\|t_{m_{k}}-x_{m_{k}}\right\| \\
& =\left\|x_{m_{k}}+\alpha_{m_{k}}\left(x_{m_{k}}-x_{m_{k}-1}\right)-\beta_{m_{k}}\left[x_{m_{k}}+\alpha_{m_{k}}\left(x_{m_{k}}-x_{m_{k}-1}\right)\right]-x_{m_{k}}\right\| \\
& \leq \alpha_{m_{k}}\left\|x_{m_{k}}-x_{m_{k}-1}\right\|+\beta_{m_{k}}\left\|x_{m_{k}}\right\|+\alpha_{m_{k}} \beta_{m_{k}}\left\|x_{m_{k}}-x_{m_{k}-1}\right\| \\
& =\beta_{m_{k}} \frac{\alpha_{m_{k}}}{\beta_{m_{k}}}\left\|x_{m_{k}}-x_{m_{k}-1}\right\|+\beta_{m_{k}}\left\|x_{m_{k}}\right\|+\beta_{m_{k}}^{2} \frac{\alpha_{m_{k}}}{\beta_{m_{k}}}\left\|x_{m_{k}}-x_{m_{k}-1}\right\|
\end{aligned}
$$


$\longrightarrow 0$.

It follows that

$$
\lim _{k \rightarrow \infty}\left\|x_{m_{k}}-x_{m_{k}+1}\right\| \leq \lim _{k \rightarrow \infty}\left\|x_{m_{k}}-t_{m_{k}}\right\|+\lim _{k \rightarrow \infty}\left\|t_{m_{k}}-x_{m_{k}+1}\right\|=0 .
$$

We have to use the same justification as in the case 1 , such that

$$
\limsup _{k \rightarrow \infty}\left\langle\varsigma^{*}, \varsigma^{*}-x_{m_{k}+1}\right\rangle \leq 0 .
$$

Now, using expressions (40) and (41), we have

$$
\begin{aligned}
& \left\|x_{m_{k}+1}-\varsigma^{*}\right\|^{2} \\
& \leq\left(1-\beta_{m_{k}}\right)\left\|x_{m_{k}}-\varsigma^{*}\right\|^{2}+\beta_{m_{k}}\left[\alpha_{m_{k}}\left\|x_{m_{k}}-x_{m_{k}-1}\right\| \frac{\alpha_{m_{k}}}{\beta_{m_{k}}}\left\|x_{m_{k}}-x_{m_{k}-1}\right\|\right. \\
& \quad+2\left(1-\beta_{m_{k}}\right)\left\|x_{m_{k}}-\varsigma^{*}\right\| \frac{\alpha_{m_{k}}}{\beta_{m_{k}}}\left\|x_{m_{k}}-x_{m_{k}-1}\right\| \\
& \left.\quad+2\left\|\varsigma^{*}\right\|\left\|t_{m_{k}}-x_{m_{k}+1}\right\|+2\left\langle\varsigma^{*}, \varsigma^{*}-x_{m_{k}+1}\right\rangle\right] \\
& \leq\left(1-\beta_{m_{k}}\right)\left\|x_{m_{k+1}}-\varsigma^{*}\right\|^{2}+\beta_{m_{k}}\left[\alpha_{m_{k}}\left\|x_{m_{k}}-x_{m_{k}-1}\right\| \frac{\alpha_{m_{k}}}{\beta_{m_{k}}}\left\|x_{m_{k}}-x_{m_{k}-1}\right\|\right. \\
& \quad+2\left(1-\beta_{m_{k}}\right)\left\|x_{m_{k}}-\varsigma^{*}\right\| \frac{\alpha_{m_{k}}}{\beta_{m_{k}}}\left\|x_{m_{k}}-x_{m_{k}-1}\right\| \\
& \left.\quad+2\left\|\varsigma^{*}\right\|\left\|t_{m_{k}}-x_{m_{k}+1}\right\|+2\left\langle\varsigma^{*}, \varsigma^{*}-x_{m_{k}+1}\right\rangle\right] .
\end{aligned}
$$

The above implies that

$$
\begin{aligned}
& \left\|x_{m_{k}+1}-\varsigma^{*}\right\|^{2} \\
& \leq\left[\alpha_{m_{k}}\left\|x_{m_{k}}-x_{m_{k}-1}\right\| \frac{\alpha_{m_{k}}}{\beta_{m_{k}}}\left\|x_{m_{k}}-x_{m_{k}-1}\right\|\right. \\
& \quad+2\left(1-\beta_{m_{k}}\right)\left\|x_{m_{k}}-\varsigma^{*}\right\| \frac{\alpha_{m_{k}}}{\beta_{m_{k}}}\left\|x_{m_{k}}-x_{m_{k}-1}\right\| \\
& \left.\quad+2\left\|\varsigma^{*}\right\|\left\|t_{m_{k}}-x_{m_{k}+1}\right\|+2\left\langle\varsigma^{*}, \varsigma^{*}-x_{m_{k}+1}\right\rangle\right] .
\end{aligned}
$$

Since $\beta_{m_{k}} \rightarrow 0$, and $\left\|x_{m_{k}}-\varsigma^{*}\right\|$ is a bounded. Thus, expressions (45) and (47), we have

$$
\left\|x_{m_{k}+1}-\varsigma^{*}\right\|^{2} \rightarrow 0, \text { as } k \rightarrow \infty .
$$

It means that

$$
\lim _{n \rightarrow \infty}\left\|x_{k}-\varsigma^{*}\right\|^{2} \leq \lim _{n \rightarrow \infty}\left\|x_{m_{k}+1}-\varsigma^{*}\right\|^{2} \leq 0 .
$$

As a consequence, $x_{n} \rightarrow \varsigma^{*}$. This will conclude the proof of the theorem. 


\section{Theoretical application}

Now, we study fixed-point problems on the basis of the following conditions:

$(\mathcal{S} 1) \mathcal{S}: \mathbb{K} \rightarrow \mathbb{K}$ is called to a $\kappa$-strict pseudo-contraction [28] upon $\mathbb{K}$ if

$$
\left\|T y_{1}-T y_{2}\right\|^{2} \leq\left\|y_{1}-y_{2}\right\|^{2}+\kappa\left\|\left(y_{1}-T y_{1}\right)-\left(y_{2}-T y_{2}\right)\right\|^{2}, \forall y_{1}, y_{2} \in \mathbb{K} ;
$$

(S2) sequentially weakly continuous on $\mathbb{K}$ if

$$
\mathcal{S}\left(y_{n}\right) \rightarrow \mathcal{S}\left(y^{*}\right) \text { for any sequence in } \mathbb{K} \text { satisfying } y_{n} \rightarrow y^{*} .
$$

If we take $\mathcal{S}$ is a $\kappa$-strict pseudo-contraction and weakly continuous then $f(x, y)=\langle x-\mathcal{S} x, y-x\rangle$ satisfies the conditions (h1)-(h4) (see [29]) and $2 c_{1}=$ $2 c_{2}=\frac{3-2 \kappa}{1-\kappa}$. As a consequence of results in Section 3 we have the following fixed point theorems.

Corollary 4.1. Assume that $\mathcal{S}: \mathbb{K} \rightarrow \mathbb{K}$ meet the criterion $(\mathcal{S} 1)$ - $(\mathcal{S} 2)$ and $\operatorname{Fix}(\mathcal{S}, \mathbb{K}) \neq \emptyset$. STEP 0: Select $\alpha>0, \chi_{0}>0, \mu \in(0,1)$ and $x_{-1}, x_{0} \in$ $\mathbb{K}$. Moreover, choose $\left\{\beta_{n}\right\} \subset(0,1)$ meet the conditions, i.e., $\lim _{n \rightarrow \infty} \beta_{n}=$ 0 and $\sum_{n}^{\infty} \beta_{n}=+\infty$.

STEP 1: Evaluate $t_{n}=\left(1-\beta_{n}\right)\left[x_{n}+\alpha_{n}\left(x_{n}-x_{n-1}\right)\right]$, where $\alpha_{n}$ modified on each iteration as follows:

$$
0 \leq \alpha_{n} \leq \hat{\alpha_{n}} \text { and } \hat{\alpha_{n}}= \begin{cases}\min \left\{\frac{\alpha}{2}, \frac{\epsilon_{n}}{\left\|x_{n}-x_{n-1}\right\|}\right\} & \text { if } x_{n} \neq x_{n-1} \\ \frac{\alpha}{2} & \text { else. }\end{cases}
$$

STEP 2: Compute

$$
\left\{\begin{array}{l}
y_{n}=P_{\mathbb{K}}\left[t_{n}-\chi_{n}\left(t_{n}-\mathcal{S}\left(t_{n}\right)\right)\right] \\
x_{n+1}=P_{\mathbb{E}_{n}}\left[t_{n}-\chi_{n}\left(y_{n}-\mathcal{S}\left(y_{n}\right)\right)\right]
\end{array}\right.
$$

where $\mathbb{E}_{n}=\left\{z \in \mathbb{E}:\left\langle\left(1-\chi_{n}\right) t_{n}+\chi_{n} \mathcal{S}\left(t_{n}\right)-y_{n}, z-y_{n}\right\rangle \leq 0\right\}$ and $\chi_{n+1}$ for next iteration is evaluated in the following way:

$$
\chi_{n+1}=\left\{\begin{array}{c}
\min \left\{\chi_{n}, \frac{\mu\left\|t_{n}-y_{n}\right\|^{2}+\mu\left\|x_{n+1}-y_{n}\right\|^{2}}{2\left\langle\left(t_{n}-y_{n}\right)-\left[\mathcal{S}\left(t_{n}\right)-\mathcal{S}\left(y_{n}\right)\right], x_{n+1}-y_{n}\right\rangle}\right\} \\
\text { if }\left\langle\left(t_{n}-y_{n}\right)-\left[\mathcal{S}\left(t_{n}\right)-\mathcal{S}\left(y_{n}\right)\right], x_{n+1}-y_{n}\right\rangle>0, \\
\chi_{n} \quad \text { else. }
\end{array}\right.
$$

Then, the sequence $\left\{x_{n}\right\}$ converges strongly to $\varsigma^{*} \in F i x(\mathcal{S}, \mathbb{K})$. 
Now, we discuss the application of our findings in the problem of classic variational inequalities [30] and the variational inequalities problem (VIP) for an operator $\mathcal{L}: \mathbb{E} \rightarrow \mathbb{E}$ is to

$$
\text { Find } \varsigma^{*} \in \mathbb{K} \text { such that }\left\langle\mathcal{L}\left(\varsigma^{*}\right), y-\varsigma^{*}\right\rangle \geq 0, \forall y \in \mathbb{K} .
$$

We assume that the following requirements have been fulfilled:

$(\mathcal{L} 1)$ Solution set of the problem $(\mathrm{VIP})$, represented by $V I(\mathcal{L}, \mathbb{K})$ is non-empty.

$(\mathcal{L} 2)$ A operator $\mathcal{L}: \mathbb{E} \rightarrow \mathbb{E}$ is called to be pseudomonotone, i.e.,

$$
\left\langle\mathcal{L}\left(y_{1}\right), y_{2}-y_{1}\right\rangle \geq 0 \Longrightarrow\left\langle\mathcal{L}\left(y_{2}\right), y_{1}-y_{2}\right\rangle \leq 0, \forall y_{1}, y_{2} \in \mathbb{K} .
$$

$(\mathcal{L} 3)$ A operator $\mathcal{L}: \mathbb{E} \rightarrow \mathbb{E}$ is said to be Lipschitz continuous with constant $L>0$, i.e., there exists $L>0$ such that

$$
\left\|\mathcal{L}\left(y_{1}\right)-\mathcal{L}\left(y_{2}\right)\right\| \leq L\left\|y_{1}-y_{2}\right\|, \forall y_{1}, y_{2} \in \mathbb{K} .
$$

$(\mathcal{L} 4)$ A operator $\mathcal{L}: \mathbb{E} \rightarrow \mathbb{E}$ is said to be sequentially weakly continuous, i.e., $\left\{\mathcal{L}\left(y_{n}\right)\right\}$ converges weakly to $\mathcal{L}(y)$ for each sequence $\left\{y_{n}\right\}$ converges weakly to $y$.

Corollary 4.2. Suppose that $\mathcal{L}: \mathbb{K} \rightarrow \mathbb{E}$ is a function meet the items $(\mathcal{L} 1)$ $(\mathcal{L} 4)$. Step 0: Select $\alpha>0, \chi_{0}>0, \mu \in(0,1)$ and $x_{-1}, x_{0} \in \mathbb{K}$. Moreover, choose $\left\{\beta_{n}\right\} \subset(0,1)$ meet the conditions, i.e., $\lim _{n \rightarrow \infty} \beta_{n}=0$ and $\sum_{n}^{\infty} \beta_{n}=$ $+\infty$.

SteP 1: Evaluate $t_{n}=\left(1-\beta_{n}\right)\left[x_{n}+\alpha_{n}\left(x_{n}-x_{n-1}\right)\right]$, where $\alpha_{n}$ modified one each iteration as follows:

$$
0 \leq \alpha_{n} \leq \hat{\alpha_{n}} \text { and } \hat{\alpha_{n}}= \begin{cases}\min \left\{\frac{\alpha}{2}, \frac{\epsilon_{n}}{\left\|x_{n}-x_{n-1}\right\|}\right\} & \text { if } x_{n} \neq x_{n-1}, \\ \frac{\alpha}{2} & \text { else. }\end{cases}
$$

SteP 2: Evaluate

$$
\left\{\begin{array}{l}
y_{n}=P_{\mathbb{K}}\left(t_{n}-\chi_{n} \mathcal{L}\left(t_{n}\right)\right), \\
x_{n+1}=P_{\mathbb{E}_{n}}\left(t_{n}-\chi_{n} \mathcal{L}\left(y_{n}\right)\right),
\end{array}\right.
$$

where $\mathbb{E}_{n}=\left\{z \in \mathbb{E}:\left\langle t_{n}-\chi_{n} \mathcal{L}\left(t_{n}\right)-y_{n}, z-y_{n}\right\rangle \leq 0\right\}$ and $\chi_{n+1}$ for next iteration is evaluated in the following manner:

$$
\chi_{n+1}=\left\{\begin{array}{l}
\min \left\{\chi_{n}, \frac{\mu\left\|t_{n}-y_{n}\right\|^{2}+\mu\left\|x_{n+1}-y_{n}\right\|^{2}}{2\left\langle\mathcal{L}\left(t_{n}\right)-\mathcal{L}\left(y_{n}\right), x_{n+1}-y_{n}\right\rangle}\right\} \\
\text { if }\left\langle\mathcal{L}\left(t_{n}\right)-\mathcal{L}\left(y_{n}\right), x_{n+1}-y_{n}\right\rangle>0 \\
\chi_{n} \quad \text { else. }
\end{array}\right.
$$


Then, the sequences $\left\{x_{n}\right\}$ converge strongly to $\varsigma^{*} \in V I(\mathcal{L}, \mathbb{K})$.

\section{Numerical illustrations}

In this section, we give some numerical example to show the implementation of our proposed method. Information about the control parameters considered as follows: (1) Algorithm 3.2 in [18] (EgA-1): $\chi=\frac{1}{3 c_{1}}, \alpha_{n}=\frac{1}{5(n+2)}$, error term $=$ $\left\|x_{n}-y_{n}\right\|^{2}$. (2) Algorithm 3 in [20] (EgA-2): $\chi=\frac{1}{3 c_{1}}, \theta=0.40, \epsilon_{n}=\frac{1}{(n+1)^{2}}, \gamma_{n}=$ $\frac{1}{5(n+2)}, \beta_{n}=\frac{5}{10}\left(1-\gamma_{n}\right)$, error term $=\left\|t_{n}-y_{n}\right\|^{2}$. (3) Algorithm 1 (EgA-3): $\chi_{0}=$ $0.01, \mu=0.40, \alpha=0.40, \epsilon_{n}=\frac{1}{(n+1)^{2}}, \beta_{n}=\frac{1}{20(n+2)}$, error term $=\left\|t_{n}-y_{n}\right\|^{2}$.

Example 5.1. Let a bifunction $f$ is defined as follows:

$$
f(x, y)=\langle P x+Q y+c, y-x\rangle, x, y \in \mathbb{K},
$$

while $c \in \mathbb{R}^{N}$ and matrices $P, Q$ are of order $N$. A matrix $P$ is symmetric positive semi-definite and the matrix $Q-P$ is symmetric negative semidefinite and Lipschitz-type constants $c_{1}=c_{2}=\frac{1}{2}\|P-Q\|$ (see [17] for details). The feasible set $C \subset \mathbb{R}^{N}$ is defined by

$$
\mathbb{K}:=\left\{x \in \mathbb{R}^{N}:-10 \leq x_{i} \leq 10\right\} .
$$

\section{Conclusion}

We have studied a extragradient-type method for determining the numerical solution of equilibrium problem in real Hilbert spaces and also prove that the generated sequence converges strongly to the solution. Numerical conclusions were drawn to explain the numerical effectiveness of our algorithms in contrast to other methods. These experimental studies showed that inertial impact improve the efficiency of the iterative sequence in this context.

\section{Acknowledgements}

This project was supported by Rajamangala University of Technology Phra Nakhon (RMUTP). 


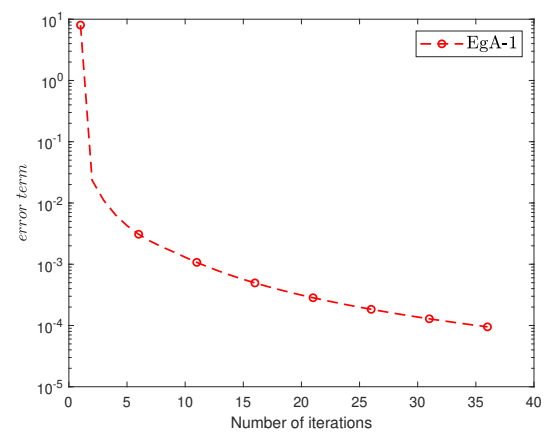

(a)

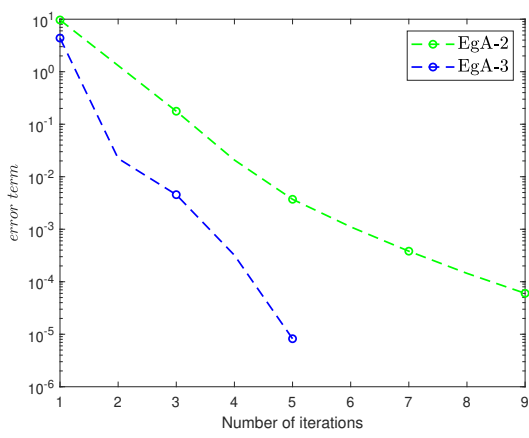

(b)

Figure 1: Example 5.1: Numerical efficiency comparison when $N=$ 2 and the number of iterations are 36, 9, 5 and elapsed time are $0.776432,0.217280,0.094933$, respectively.

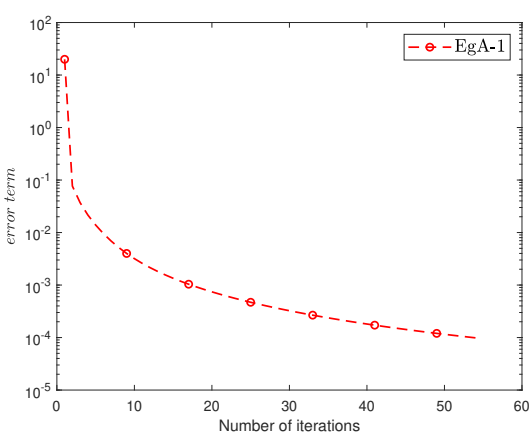

(a)

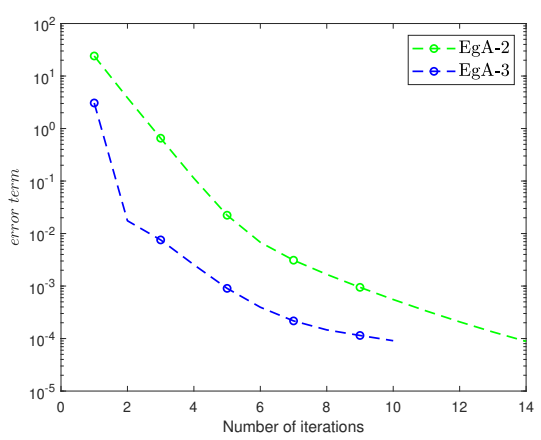

(b)

Figure 2: Example 5.1: Numerical efficiency comparison when $N=5$ and the number of iterations are 54, 14, 10 and elapsed time are $1.208463,0.366344,0.166927$, respectively.

\section{References}

[1] L. Muu and W. Oettli, Convergence of an adaptive penalty scheme for finding constrained equilibria, Nonlinear Analysis. Theory, Methods \& Applications, 18, No 12 (1992), 1159-1166.

[2] E. Blum, From optimization and variational inequalities to equilibrium 


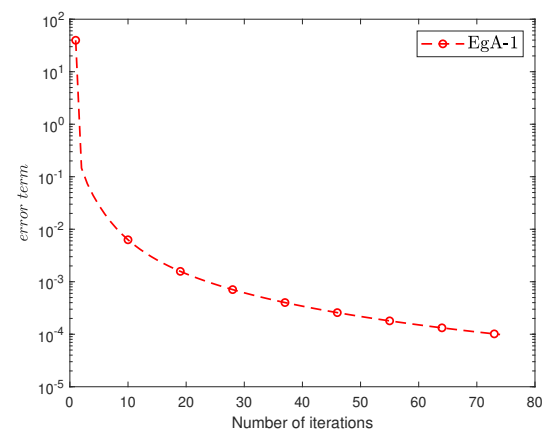

(a)

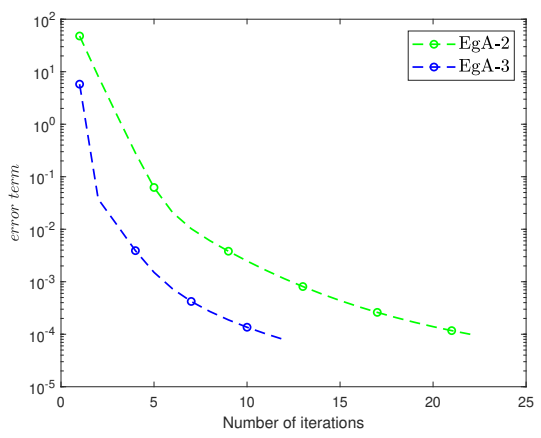

(b)

Figure 3: Example 5.1: Numerical efficiency comparison when $N=$ 10 and the number of iterations are 74, 22, 12 and elapsed time are 1.533687, 0.515103, 0.192677, respectively.

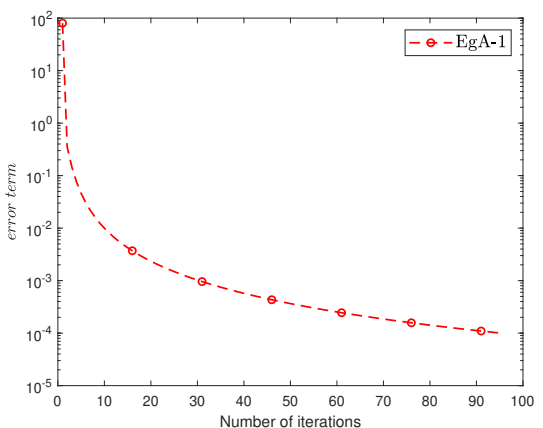

(a)

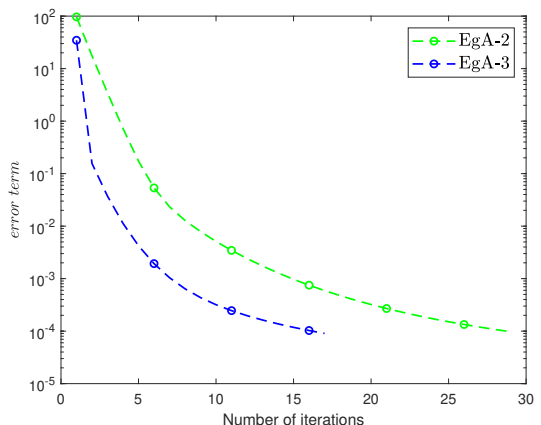

(b)

Figure 4: Example 5.1: Numerical efficiency comparison when $N=$ 20 and the number of iterations are 96, 29, 17 and elapsed time are $2.226920,0.796662,0.279478$, respectively.

problems, Math. Student, 63, No 1 (1994), 123-145.

[3] K. Fan, A minimax inequality and applications, Inequalities, 3 (1972), 103-113.

[4] M. Bianchi and S. Schaible, Generalized monotone bifunctions and equi- 


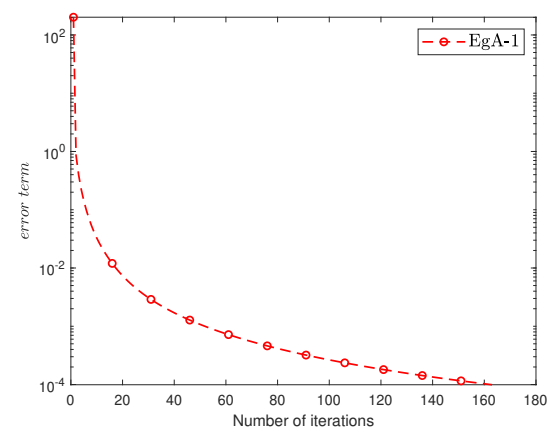

(a)

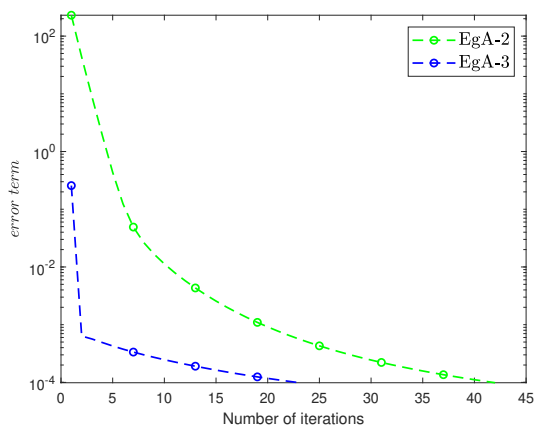

(b)

Figure 5: Example 5.1: Numerical efficiency comparison when $N=$ 50 and the number of iterations are 233, 72,48 and elapsed time are $8.333772,2.548001,1.018528$, respectively.

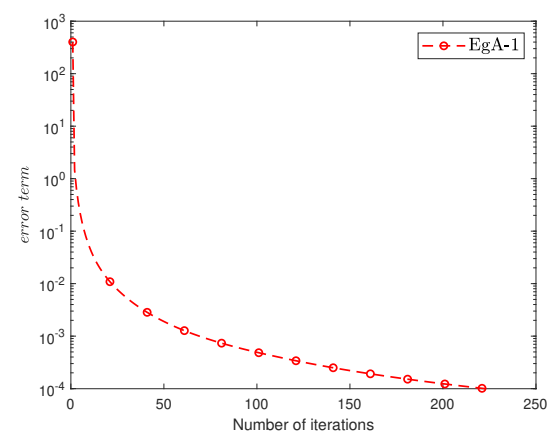

(a)

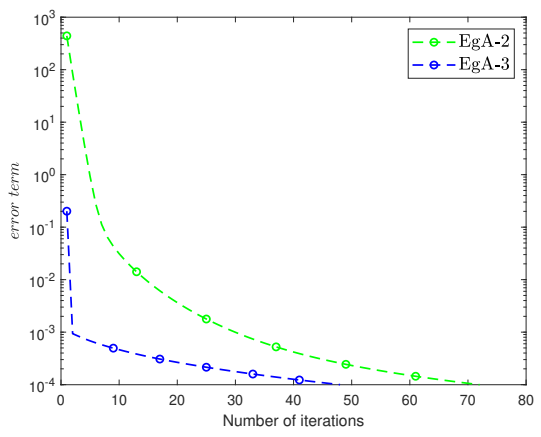

(b)

Figure 6: Example 5.1: Numerical efficiency comparison when $N=$ 100 and the number of iterations are 233, 72, 48 and elapsed time are $8.333772,2.548001,1.018528$, respectively.

librium problems, Journal of Optimization Theory and Applications, 90, No 1 (1996), 31-43.

[5] G. Mastroeni, On auxiliary principle for equilibrium problems, In: Nonconvex Optimization and Its Applications, Springer, New York (2003), 289298. 


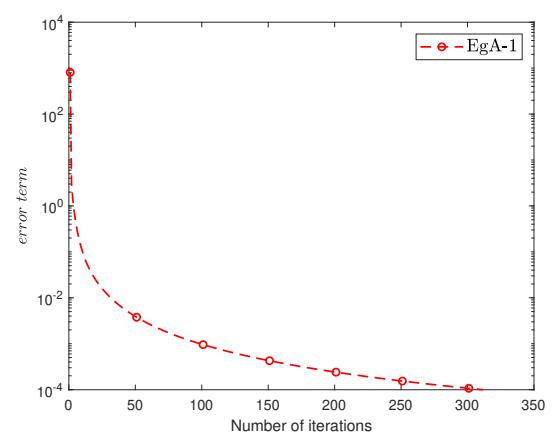

(a)

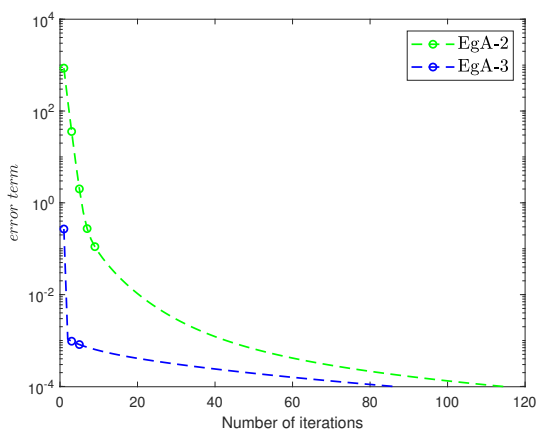

(b)

Figure 7: Example 5.1: Numerical efficiency comparison when $N=$ 100 and the number of iterations are 312, 115, 87 and elapsed time are $17.5202591,6.9271499,3.011774$, respectively.

[6] G. Bigi, M. Castellani, M. Pappalardo and M. Passacantando, Existence and solution methods for equilibria, European Journal of Operational Research, 227, No 1 (2013), 1-11.

[7] I. Konnov, Equilibrium Models and Variational Inequalities, Elsevier, Amsterdam (2007).

[8] P. L. Combettes and S. A. Hirstoaga, Equilibrium programming in Hilbert spaces, Journal of Nonlinear Convex Analysis, 6, No 1 (2005), 117-136.

[9] A. N. Iusem, G. Kassay and W. Sosa, On certain conditions for the existence of solutions of equilibrium problems, Mathematical Programming, 116, No 1 (2007), 259-273.

[10] H. Rehman, P. Kumam, Y. J. Cho and P. Yordsorn, Weak convergence of explicit extragradient algorithms for solving equilibrium problems, Journal of Inequalities and Applications, 2019, No 1 (2019); doi: 10.1186/s13660019-2233-1.

[11] H. Rehman, P. Kumam, Y. J. Cho, Y. I. Suleiman and W. Kumam, Modified Popov's explicit iterative algorithms for solving pseudomonotone equilibrium problems, Optimization Methods and Software, 36, No 1 (2020), 82-113. 
[12] H. Rehman, P. Kumam, A. B. Abubakar and Y. J. Cho, The extragradient algorithm with inertial effects extended to equilibrium problems, Computational and Applied Mathematics, 39, No 2 (2020); doi: 10.1007/s40314020-1093-0.

[13] H. Rehman, P. Kumam, I. K. Argyros and N. A. Alreshidi, Modified proximal-like extragradient methods for two classes of equilibrium problems in Hilbert spaces with applications, Computational and Applied Mathematics, 40, No 2 (2021); doi: 10.1007/s40314-020-01385-3.

[14] H. Rehman, P. Kumam, W. Kumam, M. Shutaywi and W. Jirakitpuwapat, The inertial sub-gradient extra-gradient method for a class of pseudomonotone equilibrium problems, Symmetry, 12, No 3 (2020), 463.

[15] G. Korpelevich, The extragradient method for finding saddle points and other problems, Matecon, 12, No 1 (1976), 747-756.

[16] S. D. Flåm and A. S. Antipin, Equilibrium programming using proximallike algorithms, Mathematical Programming, 78, No 1 (1996), 29-41.

[17] D. Q. Tran, M. L. Dung and V. H. Nguyen, Extragradient algorithms extended to equilibrium problems Optimization, 57, No 6 (2008), 749-776.

[18] D. V. Hieu, Halpern subgradient extragradient method extended to equilibrium problems, Revista de la Real Acad. de Ciencias Exactas, Fis. y Nat. Ser. A. Matemáticas, 111, No 3 (2017), 823-840.

[19] S. I. Lyashko and V. V. Semenov, A new two-step proximal algorithm of solving the problem of equilibrium programming, In: Optimization and Its Applications in Control and Data Sciences, Springer Intern. Publ., Cham (2016), 315-325.

[20] N. T. Vinh and L. D. Muu, Inertial extragradient algorithms for solving equilibrium problems, Acta Mathematica Vietnamica, 44, No 3 (2019), 639-663.

[21] Y. Censor, A. Gibali and S. Reich, The subgradient extragradient method for solving variational inequalities in Hilbert space, Journal of Optimization Theory and Applications, 148, No 2 (2010), 318-335.

[22] D. V. Hieu, P. K. Quy, and L. V. Vy, Explicit iterative algorithms for solving equilibrium problems, Calcolo, 56, No 2 (2019), 1-21. 
[23] E. Kreyszig, Introductory Functional Analysis with Applications, Wiley, New York (1978).

[24] J. V. Tiel, Convex Analysis: an Introductory Text, Wiley, New York (1984).

[25] H.-K. Xu, Another control condition in an iterative method for nonexpansive mappings, Bull. of the Australian Math. Soc., 65, No 1 (2002), 109-113.

[26] P.-E. Maingé, Strong convergence of projected subgradient methods for nonsmooth and nonstrictly convex minimization, Set-Valued Analysis, 16, No 7-8 (2008), 899-912.

[27] P. L. C. a. Heinz H. Bauschke, Convex Analysis and Monotone Operator Theory in Hilbert Spaces, Springer, New York (2017).

[28] F. Browder and W. Petryshyn, Construction of fixed points of nonlinear mappings in Hilbert space, Journal of Mathematical Analysis and Applications, 20, No 2(1967), 197-228.

[29] S. Wang, Y. Zhang, P. Ping, Y. Cho and H. Guo, New extragradient methods with non-convex combination for pseudomonotone equilibrium problems with applications in Hilbert spaces, Filomat, 33, No 6 (2019), 1677-1693.

[30] G. Stampacchia, Formes bilinaires coercitives sur les ensembles convexes, Comptes Rendus Hebdomadaires des Sceances de l'Académie des Sciences, 258, No 18 (1964), 4413. 
